\title{
Individual Differences in Assimilation Resistance and Affective Responses in Problem Solving
}

\author{
Pieter M. Kroonenberg \\ Leiden University \\ Conrad W. Snyder, Jr. \\ University of Queensland
}

Data on problem solving collected within the framework of Eckblad's $(198$ (b) cognitive theory of affect are analyzed with three-mode principal component analysis.] Eckblad's theory contends that affect is mediated by cognitive schemes. When schemes are inadequate for input assimilation, the resistances call forth affects with respect to the events. In this study, the task information load was varied to provide different levels of resistance. Data comprised 6 judgment scales (Pleasant-Unpleasant, Interesting-Boring,Comfortable-Uncomfortable, ComplexSimple, Varied-Monotone, and Confused-Clear) by 8 problem solving tasks (ordered a priori by information load) by 32 thinteen-year-old boys. The general results were consistent with the hypotheses of curvex scale relationships and joint scale-task fan-shaped vector configurations, but the fit was modest and vulnerable to other interpretations. Although many of the problems were accommodated by Eckblad's general scheme theory, it is suggested that assimilation resistance must be more rigorously operationalized to afford greater insight into the correspondence between cognition and affect. (In general, this study illustrates the effectiveness of the threemode principal component analysis (TUCKALS) method for the assessment of the nomothetic validity of a theoretical framework as it pertains to within and across person variation.

Behaviour, thus conceived in terms of functional interaction, presupposes two essential and closely interdependent aspects: an affective aspect and a cognitive aspect. (Piaget, 1950, p. 4)

Berlyne and his colleagues for many years sought to explicate the relationships between structural aspects and aesthetic reactions to the environment (e.g., Berlyne, 1971, 1974). Combining the traditional methodological S-R behaviorist perspective with the Piagetian cognitive notions, Berlyne extensively investigated

Thanks are extended to Gudrun Eckblad for discussions about her theory, and for the use of her data. We also thank Richard A. Harshman for his extensive and detailed review of the three-mode analyses. C.W. Snyder is presently contracted by Howard University as the Evaluation Advisor to the Ministry of Education, Gaborone, Botswana (Africa).

Requests for reprints should be addressed to P.M. Kroonenberg, Faculty of Social Sciences, Leiden University, P. O. Box 9507, 2300 RA Leiden, The Netherlands. 
affective response patterns to variations in the structural and formal attributes of objects and figures. Three response dimensions and their relationships received particular attention: Complexity, Interestingness, and Pleasantness. Complexity was linked to an Uncertainty dimension which denoted the stimulus properties that mobilized arousal and its concomitant motivational processes. Arousal reduction in the face of high complexity was designated Interesting. Arousal boost under conditions of minimal complexity was designated Pleasant. In empirical studies, hedonic tone (represented by scales like Pleasant, Good, Beautiful) was nearly always found. Uncertainty (represented by scales like Complex, Indefinite, Disorderly) was also a reliable dimension. However, Interestingness proved problematic, behaving erratically from study to study.

An alternative framework which resolved some of the observed inconsistencies in the Berlyne empirical results, was proposed by Eckblad (1978; 1980; 1981b). Within this framework, also strongly influenced by Piagetian notions, she argued that affective responses were not mediated by arousal but by features of input assimilation. The mediating variable was termed Assimilation Resistance (AR) and related to both the ambiguity presented by the stimulus to perception and the cognitive capacity of the individual to incorporate the information in available schemes. No assimilation resistance is experienced if the individual has available the appropriate cognitive structure or system of schemes to understand and anticipate the (familiar) event or object. If some discrepancy between the event and the best-available scheme is experienced, then the individual changes his/her scheme structure, in order to adapt to the new situation. This can happen whenever the stimuli are new, unexpected, incongruous, or complex. The relationship between assimilation resistance and affect can be likened to the relationship between the continuous dimension of the wavelength of light and the subjective experience of an invariant order to qualitatively different colors in the spectrum of light. Affects remain distinct qualitative orientations of the individual but are linked to experiences along the continuous dimension of assimilation resistance.

In 1981(a), Eckblad analyzed data from a problem solving experiment that were gathered to test her theory about assimilation resistance and affective responses in tasks of increasing complexity. The data, which will be called the Eckblad 1981 data, comprised the responses of 32 students on 6 rating scales designed to judge the affective reactions to 8 pattern guessing tasks. Eckblad analyzed these data by performing a principal component analysis on the 256 task/subject combinations by 6 scales matrix. With respect to this analysis, Eckblad remarks that the

... procedure has the weakness that the data matrix for the component analysis contains more than one row for each subject (namely one row for each task/subject 
combination). It is not easy to judge the consequences of treating all units as equally independent in this manner, when in reality there is both within and between subject variance in the matrix. This problem, which could have been avoided by averaging ratings across subjects before the analysis, is however a price that must be paid in order to obtain a more important methodological advantage: An individual index of A(ssimilation) R(esistance). (Eckblad, 1981a, p. 3)

A way of avoiding the price Eckblad had to pay is to use the more complicated technique of three-mode principal component analysis (Kroonenberg, 1983a; Kroonenberg \& de Leeuw, 1980; Tucker, 1966, 1972). In Snyder (1988), a primarily methodological paper, a first attempt was made to apply three-mode techniques to the Eckblad 1981 data, using in addition Harshman's PARAFAC/ CANDECOMP model (Harshman \& Lundy, 1984a) and McDonald's Invariant Factors model (McDonald, 1984). However, this time the price to pay was an increasing complexity of analysis and interpretation, which also has its uncomfortable aspects.

In the present paper, as yet unpublished data collected by Eckblad in 1985 (referred to as the Eckblad 1985 data) are investigated. They were produced by 32 thirteen-year-old boys who were presented the same tasks and scales as the students in Eckblad (1981a), and for which the same procedures were followed as described there. In brief (paraphrasing Eckblad, 1981a, p. 13), each subject had to solve 8 tasks of varying complexity. The tasks involve the guessing of the color, black or white, of each cell in a quadratic display of $8 \times 8$ cells. The correct value of each cell is exposed on a computer screen after the subject's guess and stays on the screen, building up the correct pattern as the guessing proceeds. The pattern of black and white cells varies from very simple and regular in some tasks to complex but orderly arrangements, or more or less random distributions (see Eckblad, 1981 a, Figure 2). After each task is finished, the subject rates it on the six scales (see Table 1). For each of the eight levels of a priori AR, there were four parallel versions of tasks. Each subject had to judge one of the four parallel versions for each AR level.

It is the aim to present a substantive and methodological analysis of these data. Besides replicating Eckblad's previous analysis for the new sample to establish comparability of the two samples, individual differences between the boys were studied using three-mode component techniques. In contrast with the more encompassing assessment by Eckblad (1981a), we restrict ourselves here to the 32 (boys) by 6 (scales) by 8 (tasks) data, and we do not include other variables in the discussion. That is, we are investigating these data on their own in terms of the hypotheses derived from Eckblad's total theory. Further analyses including other performance variables will be published elsewhere by Eckblad herself. 
The presentation contains four parts. First, we discuss the research questions, the nature of the data, and the preprocessing necessary for the analysis proper. Next, we present the standard Eckblad analysis of such data, followed by the three-mode analysis, and finally, substantive implications are considered.

\section{Research Questions and Data Preprocessing}

The basic assumption in the theory underlying these data is that objective stimulus complexity is closely correlated with the assimilation resistance (AR) the stimulus calls forth in an individual. Easy problems evoke far less resistance when assimilated by an individual than complex problems for which their cognitive system has no set schemes. A further assumption is that AR is coordinated with a spectrum of affective response. The affective response is assumed to vary from Boredom at very low levels of AR via mastery, Pleasure and Interest at intermediate levels of AR, to Irritation and Confusion at very high levels. Note that for the analyses to follow, it is essential to keep in mind the distinction between the objective (a priori) Complexity and the subjective Complexity, which is measured by a rating scale. To what extent tasks will call forth assimilation resistance depends on both the subjects competence in solving a problem (subjective Complexity) and the objective Complexity of a task.

From Eckblad's discussion of AR (1981a, p. 2), the data should show the following characteristics:

a. The order of the scales will be as indicated in Table 1, and will be spread in a fan-like configuration in the scale component space.

b. Ratings on Pleasant, Interesting, and Complex will form singlepeaked preference functions with the peaks located at increasing levels of AR respectively.

c. The stimuli (tasks) will be represented in the component space in their AR order in a more or less circular curve around the origin, and will also be ordered according to their Objective Complexity.

d. The correlation matrix will show a curvex pattern (Eckblad, 1980, 1981a), where a curvex pattern resembles a Guttman 'simplex' pattern, except that the low (and zero) correlations are due to the non-linearity in the preference functions rather than weak (or null) relationships.

All these predictions were confirmed in her 1981 study.

A crucial assumption is that "fundamentally the same structure is present in each subject's data, and that they differ only in location of the tasks in relation to the scale vectors" (Eckblad, 1981a, p. 22), with which she means that, for instance, the rank order of the tasks on AR should be the same for all subjects, except for noise. (The extent to which this is true in the present data may be gauged from Table 6). It seems to us that a consequence of this assumption is 
that in order to find a simultaneous solution for all subjects, it is necessary to center each scale for each subject separately so that all tasks for each subject are in deviation scores from the subject's average score of the tasks on that scale. This is what Harshman and Lundy (1984b) call "centering over tasks" or "centering on the task mode," and which is the type of centering they standardly recommend for three-mode analyses. For comparison with Eckblad (1981a) we initially do not carry out this centering in the regular principal component analysis (which is used primarily for comparison with the original Eckblad study), but for the three-mode analyses this centering strategy is used.

A related question is whether the scales should be standardized to eliminate differences in variation (=sum of squares) between scales. The scale standardization is performed automatically in Eckblad's principal component analysis. For the three-mode analyses we have not standardized the scales, because we felt that differences in variation between scales were meaningful and should be included in the analysis. Comparison with a standardized analysis showed, however, that the decision was not vitally important for the results. Also, subjects were left unstandardized, primarily because we have no knowledge or intuition what the scale differences between the subjects mean. Whether, for instance, the differences were merely a matter of response style or indicative of something more fundamental (for a discussion in a comparable problem, see Van der Kloot, Kroonenberg, \& Bakker, 1985). Note, by the way, that the preprocessing of the data is different from that of Snyder (1988) for the Eckblad (1981a) data; he standardized both subjects and scales.

\section{Principal Component Analysis on Subject/Task Units}

As in Eckblad (1981a), a principal component analysis (BMDP4M, Dixon, 1981) was performed on the ratings for the 256 units on the six rating scales (Table 1). The first two components accounted for $70 \%$ of the variance, compared to 78\% for Eckblad's older students' sample. Again the component loadings (called weights by Eckblad) show a fan-shaped configuration of approximately 180 degrees (going from Clear to Complex) compared to 170 degrees in Eckblad (1981a), and the vectors are located along the fan in the order predicted by Eckblad (Figure 1). The angle between Pleasant and Complex is 107 degrees, between Interesting and Complex 85 degrees. (These values were 105 and 75 in Eckblad, 1980; 103 and 72 in Eckblad, 1981a). The configuration is, however, rotated over about 41 degrees compared to the 1981 results, indicating a higher concentration of units in the Interesting/Pleasant direction. Thus, higher values were especially given on those scales for the early tasks, and low values on Task 8 . To investigate the similarity with the 1981 data the present solution was orthonormally rotated to optimal agreement. 
Table 1

- Principle Component Analysis of 256 Task/Subject Units on 6 Rating Scales

Correlation Matrices

\begin{tabular}{|c|c|c|c|c|c|c|c|c|c|c|c|}
\hline \multicolumn{6}{|c|}{ Eckblad 1981} & \multicolumn{6}{|c|}{ Eckblad 1985} \\
\hline 1 & 2 & 3 & 4 & 5 & 6 & 1 & 2 & 3 & 4 & 5 & 6 \\
\hline 100 & & & & & & 100 & & & & & \\
\hline 68 & 100 & & & & & 45 & 100 & & & & \\
\hline 24 & 50 & 100 & & & & 01 & 19 & 100 & & & \\
\hline-13 & 18 & 46 & 100 & & & -12 & -05 & 64 & 100 & & \\
\hline-47 & -14 & 20 & 46 & 100 & & 20 & -00 & 51 & 55 & 100 & \\
\hline-83 & -60 & -10 & 25 & 53 & 100 & -49 & -46 & 08 & 34 & 21 & 100 \\
\hline
\end{tabular}

Level of $\mathrm{AR}(1,2,3,4,5,6,7,8)$

a priori order

$\begin{array}{llllll}53 & 50 & -04 & -15 & -14 & -44\end{array}$

Level of AR $(1,2,3,4,7,6,5,8)$

empirical order

$\begin{array}{llllll}54 & 50 & -05 & -20 & -17 & -44\end{array}$

Principal Components

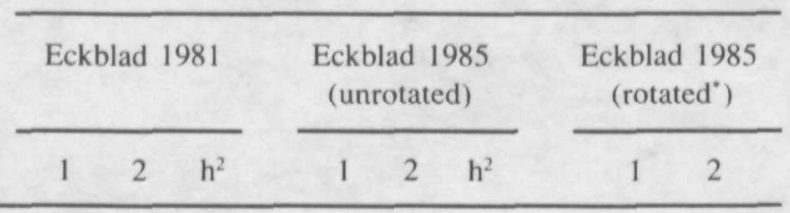

1. Complex-Simple

1. Simple-Complex

54

3. Boring-Interesting

4. Unpleasant-Pleasant

5. Uncomfortable-Comfortable

$\begin{array}{llllll}-83 & -60 & -10 & 25 & 53 & 100\end{array}$

2. Varied-Monotone

$\begin{array}{lll}93 & 04 & 87\end{array}$

$\begin{array}{lll}-52 & 61 & 64\end{array}$

$\begin{array}{ll}80 & 07\end{array}$

$\begin{array}{lll}77 & 47 & 81\end{array}$

$\begin{array}{lll}-53 & 76 & 68\end{array}$

$\begin{array}{ll}77 & 31\end{array}$

3. Interesting-Boring

$\begin{array}{lll}28 & 81 & 73\end{array}$

$\begin{array}{lll}63 & 61 & 77\end{array}$

$\begin{array}{ll}-02 & 88\end{array}$

4. Pleasant-Unpleasant

$\begin{array}{lll}-20 & 83 & 73\end{array}$

$\begin{array}{lll}80 & 35 & 77\end{array}$

$\begin{array}{ll}-32 & 81\end{array}$

5. Comfortable-Uncomfortable

$\begin{array}{lll}-60 & 58 & 70\end{array}$

$\begin{array}{lll}73 & 33 & 64\end{array}$

$\begin{array}{ll}-28 & 75\end{array}$

6. Clear-Confused

$\begin{array}{lll}-92 & 13 & 80\end{array}$

$\begin{array}{lll}65 & -52 & 68\end{array}$

$\begin{array}{rr}-83 & 09\end{array}$

\% Variance Explained

Sum Variance Explained

$46 \quad 32$

$40 \quad 30$

$35 \quad 35$

78

70

Note. Decimal points omitted. Scales are ordered a priori, 8 Congruence between rotated Eckblad 1985 and Eckblad 1981: 0.96 and 0.99, first axes and second axes, respectively (1985 solution rotated to agree optimally with 1981 solution). 
The correlation matrix for the variables (Table 1) is again a nearly perfect curvex with correlations ranging from large positive just below the diagonal to large negative in the lower left-hand corner. Compared to Eckblad (1981a) the pattern is not as strong and all but three of the correlations are lower in absolute value in the present study. Furthermore, the scales cluster more: Complex, Clear, and Varied forming one cluster; and Pleasant, Interesting, and Comfortable the other, indicating possibly less discriminatory use of the affect scales by the younger boys.

The mean component scores for each a priori level of AR were computed from the component scores of all units belonging to that level. The results are reported in Table 2 and Figure 1. According to the sector model the mean values of the levels should load along a regular curve around the origin ordered according to AR. In contrast to the students' data, the boys' data only partially correspond to the model, even with interchanging Tasks 6 and 7 as in Eckblad (1981a). In fact, with respect to subjective Complexity, Tasks 6 and 7 are rated equivalently, but in order to obtain a more or less regular curve Task 7 should appear before 6 on acount of its Pleasantness. We shall refer to the order $[1,2,3,4,7,6,5,8]$ as the empirical order of the tasks.

Table 2 also shows the mean values for each a priori level on the six variables (scales). As predicted by Eckblad, Pleasant, Interesting, and Complex reflect single-peaked preference functions, that is, each scale first increases and

Table 2

Means of Tasks on Scales and the Components per a priori AR Level

Ratings Scales

Component Values (rotated)

AR Level a

(a priori) Clear Comfortable Pleasant Interesting Varied Complex

$\mathrm{I}^{* *} \quad \mathrm{II}^{* * *}$

\begin{tabular}{rrrrrrrrr}
\hline 1 & $\mathbf{6 . 3}$ & 5.4 & 5.3 & 4.5 & 2.5 & 1.9 & -1.03 & -0.29 \\
2 & 5.8 & 5.2 & 5.5 & 5.2 & 2.9 & 2.4 & -0.74 & -0.09 \\
3 & 5.5 & 4.8 & $\mathbf{5 . 9}$ & 5.7 & 4.3 & 2.6 & -0.38 & 0.17 \\
4 & 4.8 & $\mathbf{5 . 7}$ & 5.3 & $\mathbf{5 . 8}$ & 4.8 & 3.7 & 0.08 & $\mathbf{0 . 3 6}$ \\
7 & 4.3 & 5.0 & 5.5 & 5.0 & 5.2 & 4.2 & 0.33 & 0.15 \\
6 & 4.6 & 4.8 & 5.3 & 5.1 & 4.8 & 4.4 & 0.27 & 0.05 \\
5 & 4.2 & 4.5 & 4.8 & 4.9 & 5.2 & 4.4 & 0.47 & -0.13 \\
8 & 3.3 & 4.4 & 4.3 & 4.7 & $\mathbf{6 . 0}$ & $\mathbf{5 . 3}$ & $\mathbf{1 . 0 1}$ & -0.20 \\
Overall & 4.8 & 5.0 & 5.2 & 5.1 & 4.5 & 3.6 & & \\
\hline
\end{tabular}

Note. Highest value for each column is in bold print. ${ }^{a}$ The number equals the a priori AR level and the order is the empirical order. " ANOVA between Levels: $F=24.00 ; \mathrm{df}=7,248 ; \mathrm{p}<0.01$. *** ANOVA between Levels: $F=1.56 ; \mathrm{df}=7,248 ; \mathrm{p}=0.15$. 


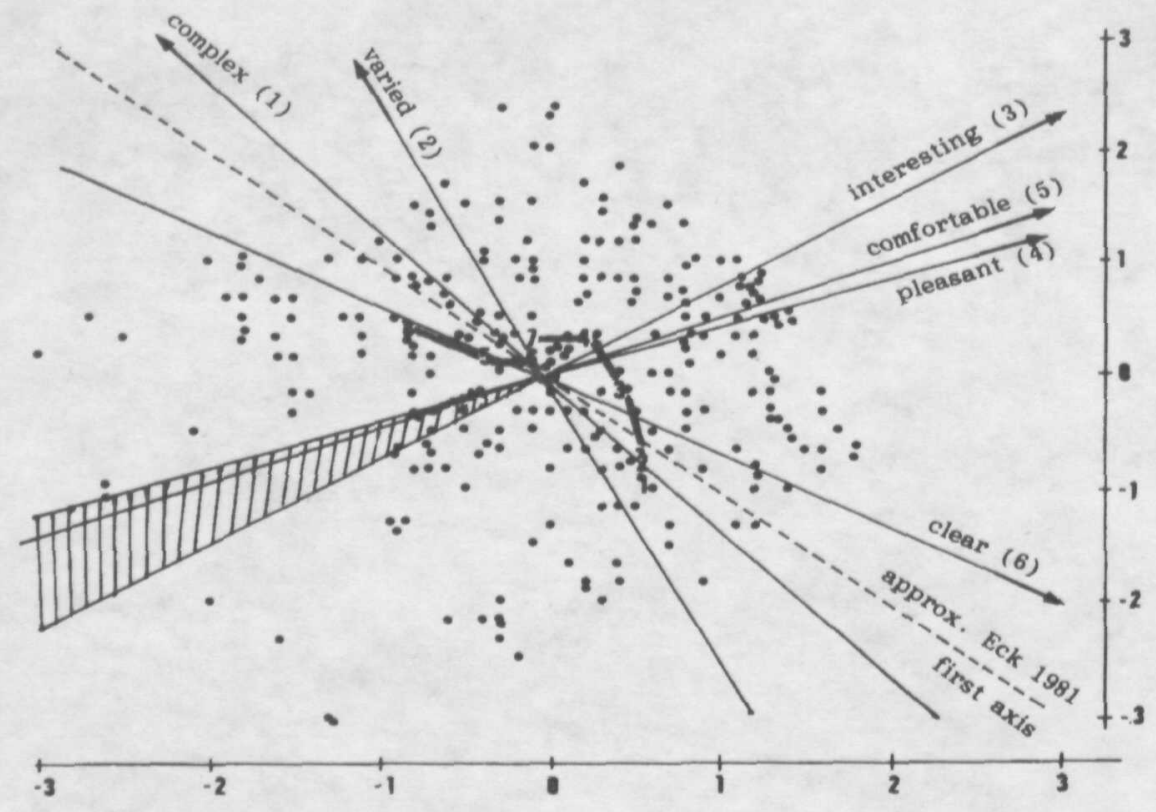

Figure 1.

Component Space from Standard Principal Component Analysis. Note: The figure contains 256 task/subject $(\bullet$ ) combinations. In the (unrotated) space, the approximate direction of the first unrotated component of Eckblad (1981a) is shown. Mean component scores for each AR level are numbered according to their $a$ priori, and connected by their empirical order. According to Eckblad's theory (1981), no (or few) subjects should be located in the hatched area.

then decreases for increasing $\mathrm{AR}$, and the peaks are located at increasingly higher levels of assimilation resistance. In a later paper, Eckblad will examine the exact shape of these preference functions and their relationships with other variables. Questions which will be examined later in this paper are (1) whether the preference functions, which are found at the aggregate level, can also be found at the individual level, and (2) to what extent it is reasonable to look at the aggregate data before sorting out the details for the individuals.

The main conclusion from the principal component analysis is that for the thirteen year-old boys the curvex correlation pattern and the fan-shaped vector configuration hold up very well, and confirm the results from earlier studies, thereby extending the applicability of Eckblad's framework to adolescents. A puzzling feature is, however, the order of the tasks, that is, the relationship between subjective and objective Complexity and/or assimilation resistance. At least two factors might play a role in this. First, the complexity most of the boys were able to handle lies somewhere between Task 4 and 5; Tasks 5, 6, and 7 were 
judged almost equally Complex, Confusing, etcetera, while Task 8 was clearly recognized as even more complex (G. Eckblad, personal communication, 1986). In other words, the wrong order is more apparent than real.

A second possibility for the deviant place of Task 5 could have been the nature of the complexity in 5 , which might not have been grasped adequately by the thirteen year-olds after the complete pattern was shown. In other words, they may have failed to recognize the regularity in the pattern (see Figure 2 in Eckblad, 1981a).

As an aside, the equivalence of the four parallel versions of the tasks within an a priori level of AR was checked via one-way MANOVAs with the scales as dependent variables and the four tasks within a level as the factor. No overall significant MANOVAs were found, and the only univariate significant effects were for Varied (AR level 6 and 7), and more importantly for Complexity (AR level 5). In particular, the third version was especially difficult (mean=5.6), and the fourth especially simple (mean $=3.0$, compared to an overall mean of 4.4 ), placing the latter at about level 3.5 and the former at level 8 (see Table 2 for the means in question). It is doubtful, however, if this could explain the deviant behavior of Task 5, as the same task was included in previous studies, and on the averages the differences seem to have little influence. The variance for that task of course will be increased. Overall, however, there is little reason to doubt the equivalences between parallel tasks within a level.

\section{Rationale for Three-Mode Analysis}

The principal component analysis on the subjects/tasks by variables has several drawbacks. In the first place such an analysis is only justified if the observations are thought of as the sum of a systematic part and a random error, and the systematic variation strongly dominates the autocorrelation in the error caused by the multiple rows due to the same subject (for an elucidating discussion of this point, see Visser, 1985, pp. 51-53). Secondly, for interpretational purposes, the uncoupling of the data from single individuals is rather inconvenient to get a good grasp of the nature of the individual differences, and similarly the task structure can only indirectly be investigated by averaging over the component scores for the tasks. Thirdly, as suggested by a reviewer, a regular component analysis uses more parameters to describe the structure in the data, and therefore might fit more error.

An additional, more subtle, question concerns the underlying structure of the data. In the above analysis it is assumed that the scales are responsible for the structure in the sense that scores on the six scales can be combined into scores on linear combinations of those scales. It is also appropriate to consider the tasks to have underlying structure (as Eckblad does) in the sense that the scores on the 
tasks may be adequately described by linear combinations of those tasks. In fact, "the hypothesis prescribing the order for tasks with respect to assimilation resistance suggests such a structure. If one accepts this view, then it is a matter of empirical verification if the task and scale structures coincide, both on the aggregate and the individual level, and one should look for techniques which do justice to this view.

In the present paper we use three-mode principal component analysis for this purpose, because with this technique it becomes possible to compute components for both scales and tasks independently. Additionally, the relative importance of combinations of scale and task components can be assessed either for each individual (via the Tucker2 model, 1972) or for components of individuals or ideal type individuals (via the Tucker3 model, 1966). It is furthermore possible to compute component scores for each subject/task unit on the scale components within the same framework, but taking the condensation on both scales and tasks into account. The technique was originally developed by Tucker (e.g., 1966; 1972), further developments have been made by Kroonenberg and de Leeuw (1980), while relatively elementary explications can be found in Kroonenberg (1983a, 1984), Levin (1965), and Snyder (1988). A bibliography is available in Kroonenberg, Snyder, and Law (1984), and an annotated bibliography can be found in Kroonenberg (1983b). The analyses reported in this paper were performed using the TUCKALS2 and TUCKALS3 programs (Kroonenberg \& Brouwer, 1985a, b), which are available through the first author.

Before presenting the results, some general remarks should be made about the assessment of the stability and the quality of the results. Furthermore, as will become apparent, there is a relatively modest fit of the model to the data, and this necessitates establishing if merely noise is being fitted. Finally, some check on the choice of the number of components was desired, especially in the light of the modest overall fit.

To start with the dimensionality, the procedure followed was designed in the spirit of Humphreys and Montanelli's (1975) Parallel Analysis Method. These authors constructed from random normal deviates a new correlation matrix, the factors of which were used to compare with the factors of the original correlation matrix in terms of explained variance. Because the probability structure of the present data and of most other three-mode data sets is unclear, it was decided to create Random data by sampling without replacement from the original data. This has the advantage of maintaining the overall sum of squares, and thus the overall size of the data without embodying any of its structure. The explained variation or sum of squares from these Random data will be compared with those of the original data to assist in choosing an adequate solution.

To assess the stability of the scale and task components under minor variations, five bootstrap samples (thus with replacement, see Efron, 1982) were taken from the set of 32 boys. Even though five such samples is not a substantial 
set, and insufficient to establish real confidence intervals, they can be effectively used to provide insight into the internal stability of the data set, as we see in the next section.

Finally, to gain a similar impression of the quality of the solution of the individuals, yet another procedure had to be used. The data from our random subjects in the Random data were used to compute core planes (according to the Tucker2 model, i.e. $\mathrm{C}_{\mathrm{k}}=\mathrm{G}^{\prime} \mathrm{Z}_{\mathrm{k}} \mathrm{H}$, in the notation of Kroonenberg \& de Leeuw, 1980) for each of them. The relative fits for all random subjects were computed and compared to those of the real subjects. In this way, it was possible to assess whether real subjects had a fit to their data, which exceeded those of the random subjects.

\section{Three-Mode Analysis of Tasks by Scales by Subjects}

Fit

Several three-mode analyses were performed with two and three components for scales, tasks, and subjects using the Tucker2 (Tucker, 1972)and Tucker3 (Tucker, 1966) models. Details of the fit (= fitted sums of squares) of these analyses are given in Table 3 . The primary conclusion from this table is that the overall fit is modest, indicating that a large part of the individual variation in the Eckblad 1985 data is not fitted by the model. Snyder's (1988) results for the Eckblad 1981 data are included as well, and even though their fit is better, still a large amount of the variation in these data goes unaccounted for. A probable cause of the modest fit might be the limited reliability of each particular rating point, or the presence of particularly unreliable subjects, but other possibilities are also considered in this paper. Furthermore, there is a difference in the centering and standardization of the data, so that the data base is not exactly the same. A similar standardization, however, does not greatly influence the threemode fit in this case (nor the overall solution for that matter), as can be seen from Table 3 by comparing the $-\mathrm{C}$ - and CSS solutions.

Also included in Table 3 is the fit of the Random data. Clearly the real data show more explained variation ( .44 versus .16 [ $2 \times 2$-solution], and .56 versus .31 [3 3 3-solution]). The size of the explained variations for the components indicate that three components for the scales and the tasks are not warranted because the third components have lower explained variations than the third random components. However, the second components are larger than the random ones, although only by a narrow margin of $2 \%$. Finally, the results of the bootstrap samples confirm the weak but reproducible second components (see also Table 4). All information thus points towards the acceptability of a $2 \times 2$-solution for the Tucker 2 model. 
Table 3

Eit of Three-Mode Analyses

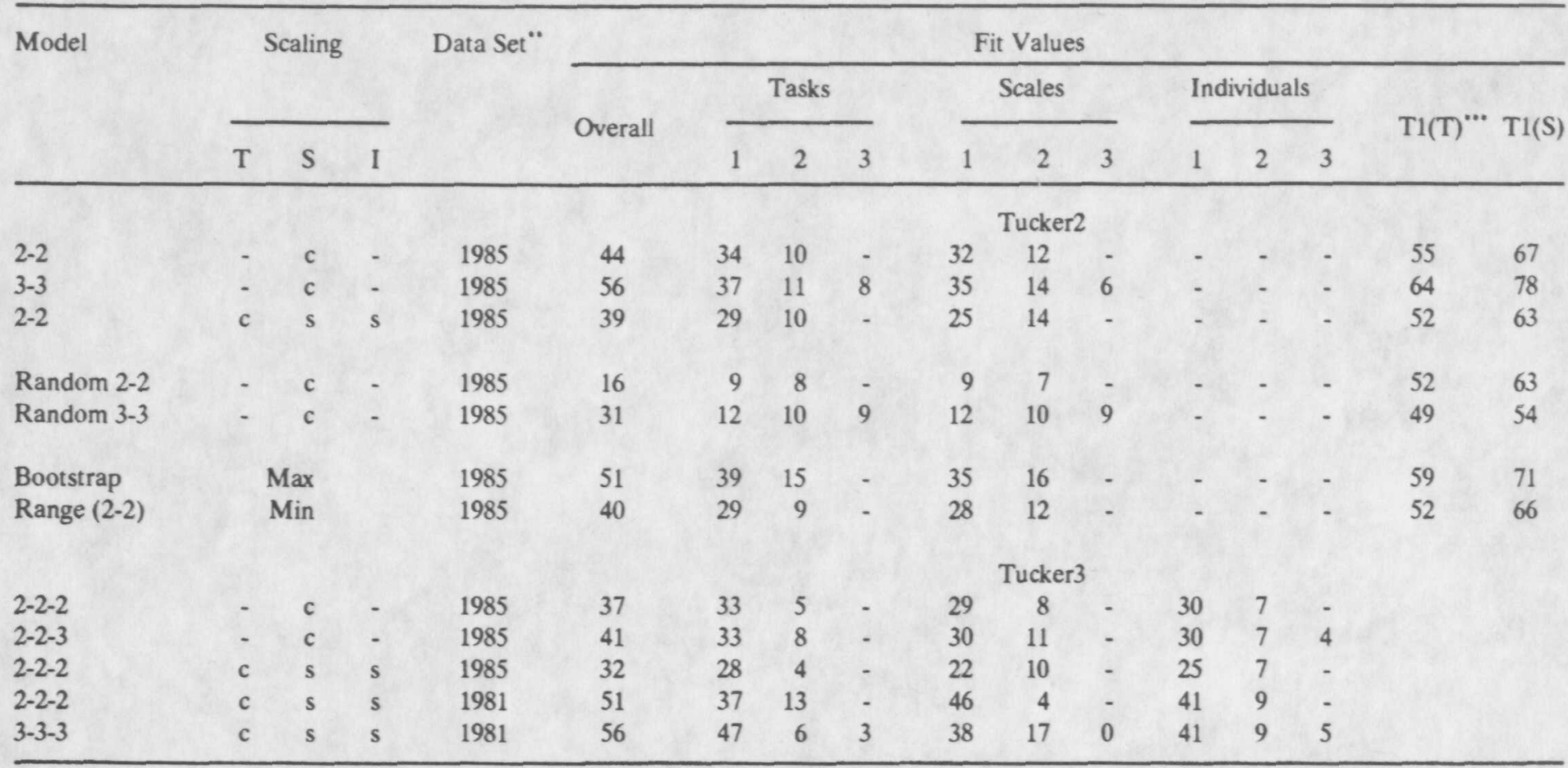

Note. An M-P-Q solution has M task (T) components, P scale (S) components, and Q individual (I) components. "-" indicates that the category is irrelevant. ' $c=$ mode centered; $s=$ mode is scaled; - = no centering or scaling. " 1981 refers to Eckbald's student data set; 1985 refers to Eckblad's new data with 13 year old boys. $\cdots \mathrm{Tl}(\mathrm{x})$ is the proportional fit of a regular PCA on the "strung-out" matrix with the same scaling as in the threemode analyses. 
The overall fit of the $2 \times 2$-solution may be compared with the fit of (twomode) principal component analyses on the same data, but strung out along one of the three modes. We performed such an analysis in the previous paragraph, but used the centering and scaling routinely employed in principal component analysis, while what we want here is to compare the three-mode analysis with two-mode analyses for identically preprocessed data. In fact, such analyses are standardly included in the TUCKALS programs (Kroonenberg \& Brouwer, $1985 \mathrm{a}, \mathrm{b})$, as they provide the starting values for the main iteration procedure, and they are, by the way, identical to the procedures in Tucker's (1966) Method I. These principal component analyses will be designated as T1(Scales) and T1(Tasks) to stay within the Lundy, Harshman, and Kruskal's (1985) parlance. With two components, both $\mathrm{T} 1(\mathrm{~S})$ and $\mathrm{T} 1(\mathrm{~T})$ achieve a better fit than the simultaneous analysis: Fit $\mathrm{T} 1(\mathrm{~T})=.55$ with a bootstrap range of .52 to .59 ; Fit $\mathrm{T} 1(\mathrm{~S})=.67$ with a bootstrap range of .66 to .71 , compared to an overall fit of .44 with a bootstrap range of .40 to .51 . Thus, each of the regular principal component analyses fit variation not fitted by the other one, and this kind of variation cannot be described by the three-mode model, as the latter only looks at the variation that the scales and tasks have in common across all individuals. Since the AR construct entails a dependency between the scale and task configurations, the loss of fit discounts the global validity of the Eckblad framework for younger students.

Note by the way that there is no great difference between the amount of explained variation between the analysis reported in the previous section (fit = .70 ) and the $\mathrm{T} 1(\mathrm{~S})$ analysis (fit $=.67$ ), and that both fall within the bootstrap range. This seems to indicate that the different preprocessing used was not of vital importance in the two analyses, since their scale components were also quite similar.

Scales and Tasks

The components for scales and tasks are given in Table 4 in a scaling comparable to that in Table 1. Clearly the scale space of the rotated principal component solution and the three-mode one are very similar. The task space is now determined directly, and it bears some resemblance to the mean component scores in Table 2. Note as mentioned before that the task and scale components are not necessarily the same, while they are in Table 1 and 2 . In the three-mode model of individual differences, each subject may combine the task and scale components in his or her own way.

The bootstrap results for the scale space show a reasonably stable first component with an ordering: Clear, (Comfortable, Pleasant), Interesting, (Varied, Complex), but with insufficient precision to separate the scales 
Table 4

Component Loadings for Tasks and Scales

Tasks

\begin{tabular}{|c|c|c|c|c|c|}
\hline \multicolumn{2}{|c|}{ Component ${ }^{*}$} & \multicolumn{4}{|c|}{ Bootstrap Range } \\
\hline \multirow[t]{2}{*}{ T1 } & \multirow[t]{2}{*}{$\mathrm{T} 2$} & \multicolumn{2}{|c|}{$\mathrm{T} 1$} & \multicolumn{2}{|c|}{$\mathrm{T} 2$} \\
\hline & & Min & $\operatorname{Max}$ & Min & Max \\
\hline-86 & -61 & -102 & -75 & -63 & -29 \\
\hline-63 & 12 & -68 & -46 & 8 & 21 \\
\hline-54 & 47 & -69 & -28 & 11 & 51 \\
\hline 2 & 22 & -15 & 15 & -07 & 47 \\
\hline 59 & -07 & 40 & 69 & -34 & 21 \\
\hline 25 & -14 & 21 & 36 & -49 & 8 \\
\hline 30 & 23 & 12 & 45 & 17 & 32 \\
\hline 87 & -22 & 82 & 94 & -33 & 04 \\
\hline 34 & 10 & 29 & 39 & 9 & 15 \\
\hline & & & & & \\
\hline
\end{tabular}

Tasks

Task 1

Task 2

Task 3

Task 4

Task 5

Task 6

Task 7

Task 8

\% Variation

Sum \% Variation

$\%$ Variation (Random Data)

9

Sum \% Variation (Random Data)

8

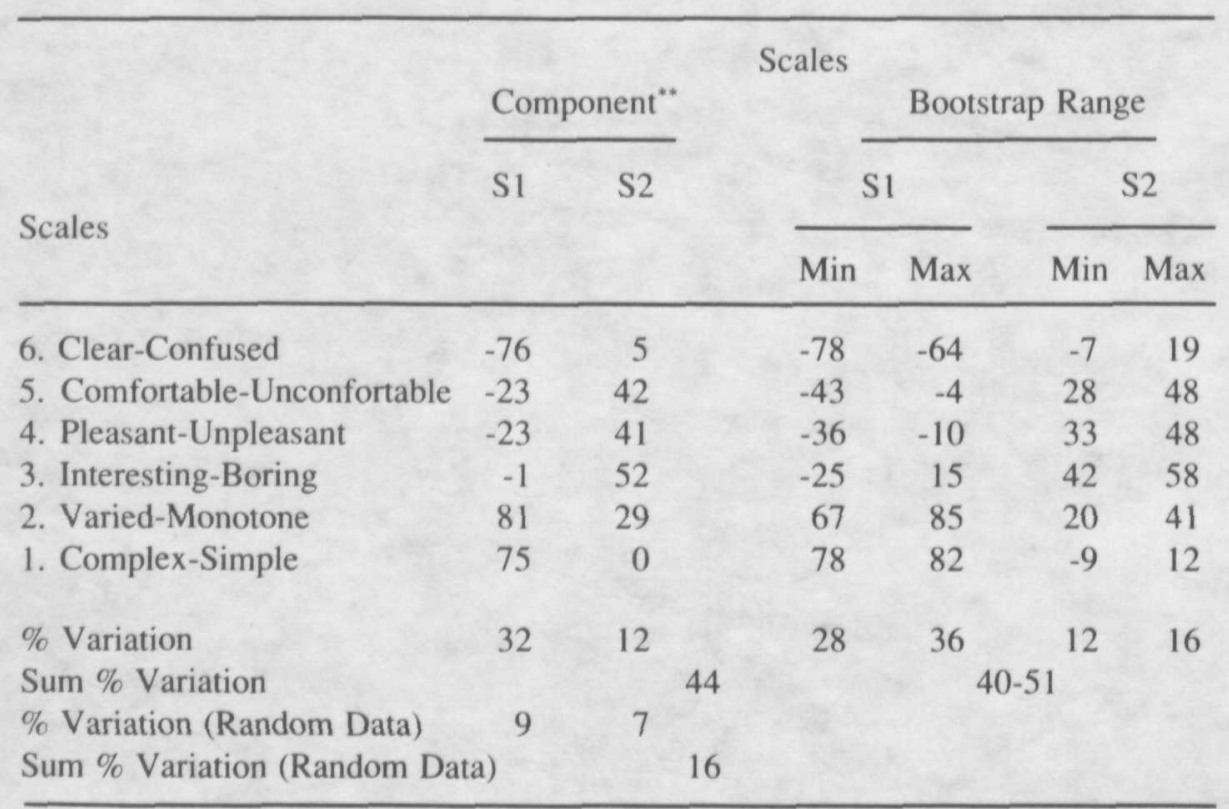

Note. Orders of Tasks and Scales are a priori ones. Variation $=$ Sum of Squares. 'Task Components are scaled to Eigenvalue $x \sqrt{8}$. " Scale Components are scaled to Eigenvalue $\mathrm{x} \sqrt{6}$. 
between brackets. The second component is less stable than the first, with wider bootstrap margins for the loadings. Here the order is (Clear, Complex), Varied, (Pleasant, Comfortable), Interesting. Thus, in two dimensions, only Pleasant and Comfortable are not separable.

The parallel results for the task space show again a reasonably stable first component which could be taken to correspond to the subjective complexity. The order of the tasks on this component is: $1,(2,3), 4,(7,6), 5,8$, with insufficient precision to separate the tasks 2 and 3 , and 7 and 6 . The second component has again wider bootstrap ranges. In fact, they are so wide that one should say that 1 and 8 are low on this component, 3, 4, and 7 are high, and 2, 5 , and 6 are around zero, but that greater precision is not available.

In her 1981a paper, Eckblad indicated that her sector model predicts "a roughly curvilinear trend around the Origin" (p. 22) for tasks, which can be observed in the present task space. She continues to state that "In the sector model, the curvilinear trend corresponds to the underlying dimension of AR, which extends from the vicinity of the optimal point for Boring to the vicinity of the optimal point for Unpleasant" (p. 22). Within the context of the model, the second task dimension should not be interpreted as a separate substantive dimension, but rather a reflection of the curvilinearity of AR with respect to complexity.

Eckblad explains the two-component representation of the hypothesized unidimensional AR construct in terms of Coomb's unfolding notions (1964; Coombs \& Kao, 1960). The affect words are applied preferentially to the range of complexity in the stimulus set. When analyzed, the result is the curvex mosaic in a two-component space, with sectors in the space defined by the pleasingness, interestingness, and judged complexity vectors. Each of the affect designations is curvilinearly related to objective complexity, with ideal points (peaks) corresponding to boredom through judged complexity at increasing levels of objective complexity. Therefore, the complete affect spectrum reflects a staggered series of single peaked functions, where the order on AR is reflected in the order of the vector termini of the fanlike bicomponent (curvex) configuration.

\section{Mutual Weights}

In Table 5 several kinds of information are given about the way the subjects consider combinations of the scale components (Complexity/Obscurity - S1 and Interestingness/Pleasantness - S2) and the Task components (T1 and T2). The subjects have been ordered with respect to the fit of the model to their data. Compared to the random subjects, we see that most real subjects have clearly a substantial Tucker 2 fit. However, eight subjects, (i.e. 6, 22, 20, 7, 9, 12, 15, and 28 ) with a fit of $.23, .23, .22, .16, .14, .14, .07$, and .07 respectively, fall 
Table 5

Relative Importance (Mutual Weights) of Task and Scale Axes for Subjects, Tucker3 Subject Components, and Core Matrix

\begin{tabular}{|c|c|c|c|c|c|c|c|c|}
\hline \multirow[t]{2}{*}{ Subject } & \multirow{2}{*}{$\begin{array}{l}\text { Plot } \\
\text { Label }\end{array}$} & \multicolumn{4}{|c|}{ Component Combinations* } & \multirow{2}{*}{$\begin{array}{c}\text { Tucker2 } \\
\text { Fit }\end{array}$} & \multicolumn{2}{|c|}{$\begin{array}{c}\text { Tucker } 3^{* *} \\
\text { Components }\end{array}$} \\
\hline & & $(\mathrm{T} 1, \mathrm{~S} 1)$ & $(\mathrm{T} 2, \mathrm{~S} 2)$ & $(\mathrm{T} 1, \mathrm{~S} 2)$ & $(\mathrm{T} 2, \mathrm{~S} 1)$ & & 1 & 2 \\
\hline 26 & Q & 56 & 9 & -5 & -6 & 72 & 26 & -0 \\
\hline 29 & $\mathrm{~T}$ & 66 & 43 & -22 & -7 & 68 & 37 & 14 \\
\hline 19 & $\mathbf{J}$ & 51 & 7 & 30 & 6 & 66 & 23 & -23 \\
\hline 18 & I & 18 & 3 & -3 & 21 & 65 & 9 & 11 \\
\hline 2 & 2 & 43 & 10 & 6 & -3 & 64 & 20 & -8 \\
\hline 1 & 1 & 47 & 9 & -11 & 2 & 62 & 22 & 7 \\
\hline 27 & $\mathrm{R}$ & 40 & -15 & 31 & -15 & 62 & 15 & -33 \\
\hline 3 & 3 & 40 & 30 & 6 & 20 & 59 & 22 & 5 \\
\hline 10 & A & 62 & -4 & 0 & -15 & 59 & 27 & -8 \\
\hline 31 & V & 19 & 3 & -61 & 25 & 59 & 10 & 60 \\
\hline 23 & $\mathrm{~N}$ & 42 & 5 & -2 & -12 & 54 & 19 & -5 \\
\hline 4 & 4 & 53 & 9 & -19 & -8 & 53 & 25 & 9 \\
\hline 8 & 8 & 49 & 10 & 13 & -2 & 52 & 23 & -13 \\
\hline 17 & $\mathrm{H}$ & 49 & 31 & -4 & 1 & 52 & 26 & 2 \\
\hline 32 & W & 40 & 21 & -5 & 12 & 52 & 20 & 9 \\
\hline 21 & $\mathrm{~L}$ & 37 & 37 & 18 & -6 & 49 & 21 & -16 \\
\hline 16 & G & 37 & 9 & -1 & 6 & 44 & 18 & 2 \\
\hline 25 & Z & 36 & 6 & 4 & 10 & 39 & 17 & -0 \\
\hline 11 & B & 26 & 11 & 17 & 3 & 36 & 13 & -14 \\
\hline 5 & 5 & 24 & 9 & -35 & 20 & 33 & 13 & 38 \\
\hline 14 & E & 29 & 10 & 3 & -22 & 29 & 14 & -12 \\
\hline 24 & $\mathrm{O}$ & 19 & -1 & 7 & 22 & 25 & 8 & 5 \\
\hline
\end{tabular}

(table continues)

within the range of the fit of the random subjects (.04 to .23$)$, and it is therefore difficult to maintain that the model contributes substantially towards the understanding of these data. (Given that we chose not to standardize subjects, the absolute sizes of the component combinations of the random subjects cannot be relied upon to screen those of real subjects, because these sizes also depend upon their total sum of squares. For instance, subject 18 has rather small mutual weights, but the fit of the data to the model is among the best; its total sum of squares after centering is only 12 compared to the average of 48 .)

The (S1, T1) combination reflects both the judged complexity and the approximate or empirical AR order, while the combination (T2, S2) indicates 
Table 5 (cont.)

\begin{tabular}{|c|c|c|c|c|c|c|c|c|}
\hline \multirow[t]{2}{*}{ Subject } & \multirow{2}{*}{$\begin{array}{l}\text { Plot } \\
\text { Label }\end{array}$} & \multicolumn{4}{|c|}{ Component Combinations ${ }^{*}$} & \multirow{2}{*}{$\begin{array}{c}\text { Tucker2 } \\
\text { Fit }\end{array}$} & \multicolumn{2}{|c|}{$\begin{array}{c}\text { Tucker } 3^{* *} \\
\text { Components }\end{array}$} \\
\hline & & $(\mathrm{T} 1, \mathrm{~S} 1)$ & $(\mathrm{T} 2, \mathrm{~S} 2)$ & $(\mathrm{T} 1, \mathrm{~S} 2)$ & $(\mathrm{T} 2, \mathrm{~S} 1)$ & & 1 & 2 \\
\hline 13 & D & 29 & -12 & 13 & -4 & 24 & 11 & -12 \\
\hline 30 & $\mathrm{U}$ & 27 & 1 & 4 & -8 & 24 & 13 & -7 \\
\hline 6 & 6 & 12 & 34 & 14 & -10 & 23 & 10 & -17 \\
\hline 22 & M & 23 & 4 & -8 & 16 & 23 & 19 & -5 \\
\hline 20 & K & 8 & -9 & -16 & 37 & 22 & 4 & 31 \\
\hline 7 & 7 & 17 & 2 & 5 & 10 & 16 & 8 & 0 \\
\hline 9 & 9 & 11 & 5 & 3 & 3 & 14 & 5 & -1 \\
\hline 12 & $\mathrm{C}$ & 13 & -6 & -4 & 20 & 14 & 6 & 10 \\
\hline 15 & $\mathrm{~F}$ & 2 & -18 & -8 & -7 & 7 & -2 & 4 \\
\hline 28 & $\mathrm{~S}$ & -5 & -1 & 7 & 12 & 7 & -2 & -9 \\
\hline \multirow{4}{*}{\multicolumn{2}{|c|}{$\begin{array}{l}\text { Average } \\
\text { Average Random } \\
\text { Data } \\
\% \text { Explained } \\
\text { Variation }\end{array}$}} & 32 & 8 & 1 & -3 & 43 & & \\
\hline & & & & & & 11 & & \\
\hline & & & & & & & & \\
\hline & & 28 & 6 & 6 & 4 & & 30 & 7 \\
\hline \multicolumn{9}{|c|}{ Range Random Data } \\
\hline & $\operatorname{Max}$ & 38 & 32 & 18 & 28 & 23 & & \\
\hline & Min & -27 & -29 & -22 & -27 & 4 & & \\
\hline \multicolumn{9}{|l|}{ T3 Core } \\
\hline Slice & & 20.4 & 6.6 & -0.7 & -0.7 & & & \\
\hline Slice & & -0.7 & -0.7 & -9.2 & 5.3 & & & \\
\hline
\end{tabular}

Note. $\quad \cdot \mathrm{T} 1 / 2=$ first/second Task component; $\mathrm{S} 1 / 2=$ first $/$ second Scale component. (See Table 4). ${ }^{*}$ Lengths of components are equal to one.

the curvature of the tasks in the direction of Interestingness/Pleasantness. Clearly most subjects use the Complexity axis as intended (apart from Task 5), and they vary greatly in their curvature. For instance, 3, 17, 21, and 29, heavily use the Pleasantness dimension in their judgments. Judging from the component combination (T1, S2), several subjects find the complexity of the tasks as expressed by the empirical order very Pleasant and Interesting (e.g., 5, 29, 31) while several others consider the opposite to be true (e.g., 19 and 27). For some subjects the contrast between Tasks 1 and 8 with mainly Tasks 3,4 , and 7 (axis T2) is associated with Complexity, as is shown by their (T2, S1) combinations. For these boys it seems that, relatively speaking, Task 1 is less and Task 3 is more 
Complex or Confused than it should be (e.g., 3, 24, 31). The reverse is true for boys 14,23, and 27, who consider Task 1 (and 2) to be more Complex than Task 3 (over and above the complexity measured by the first components). Furthermore, boys with low weights for all combinations (e.g., 28,9, 15) simply do not seem to use the structure common to most other boys.

One way to give a more condensed description of the individual differences would be to derive components for boys as well, which would entail performing a principal component analysis on the four component combinations, or what amounts to the same thing, perform a Tucker 3 analysis on the data. In Table 5 the first two subject components from a Tucker3 analysis are given as well as the corresponding slices of the three-way condensed core matrix. From the first core slice it follows that subjects loading exclusively on the first subject

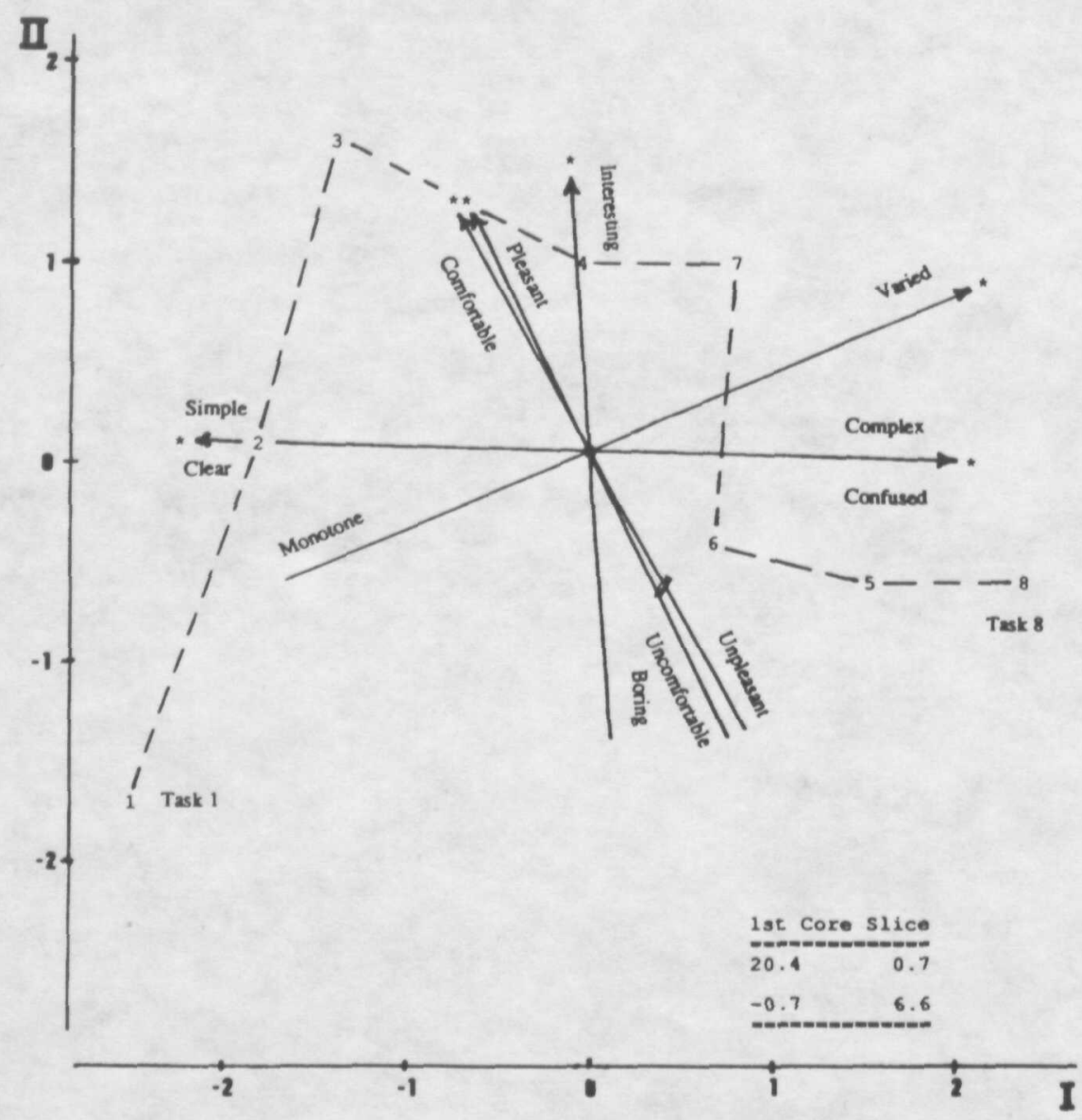

Figure 2.

Joint Plot of Scales and Tasks for First Subject Type. 
component, like for instance 26 does, weight the (T1, S1) combination (=20.4) most heavily, and also give some weight to the $(\mathrm{T} 2, \mathrm{~S} 2)$ combination $(=6.6)$, but not to the other two combinations. For a boy loading exclusively and positively on the second component (e.g., 31) the reverse is true, he weights (T1, S2) negatively $(=-9.2)$ and $(\mathrm{T} 2, \mathrm{~S} 1)$ positively $(=5.3)$, and hardly uses the other two combinations. In other words, he associates increasing Complexity of tasks with increasing Unpleasantness and Boredom, and the contrast between begin/ end tasks and middle tasks with Complexity and Confusion. From the subject components we may deduce that most boys are a mixture of two types described above in that they load more or less heavily on both subject components. In Figures 2 and 3 the relationships between scales and tasks are given for each of the two subject types.

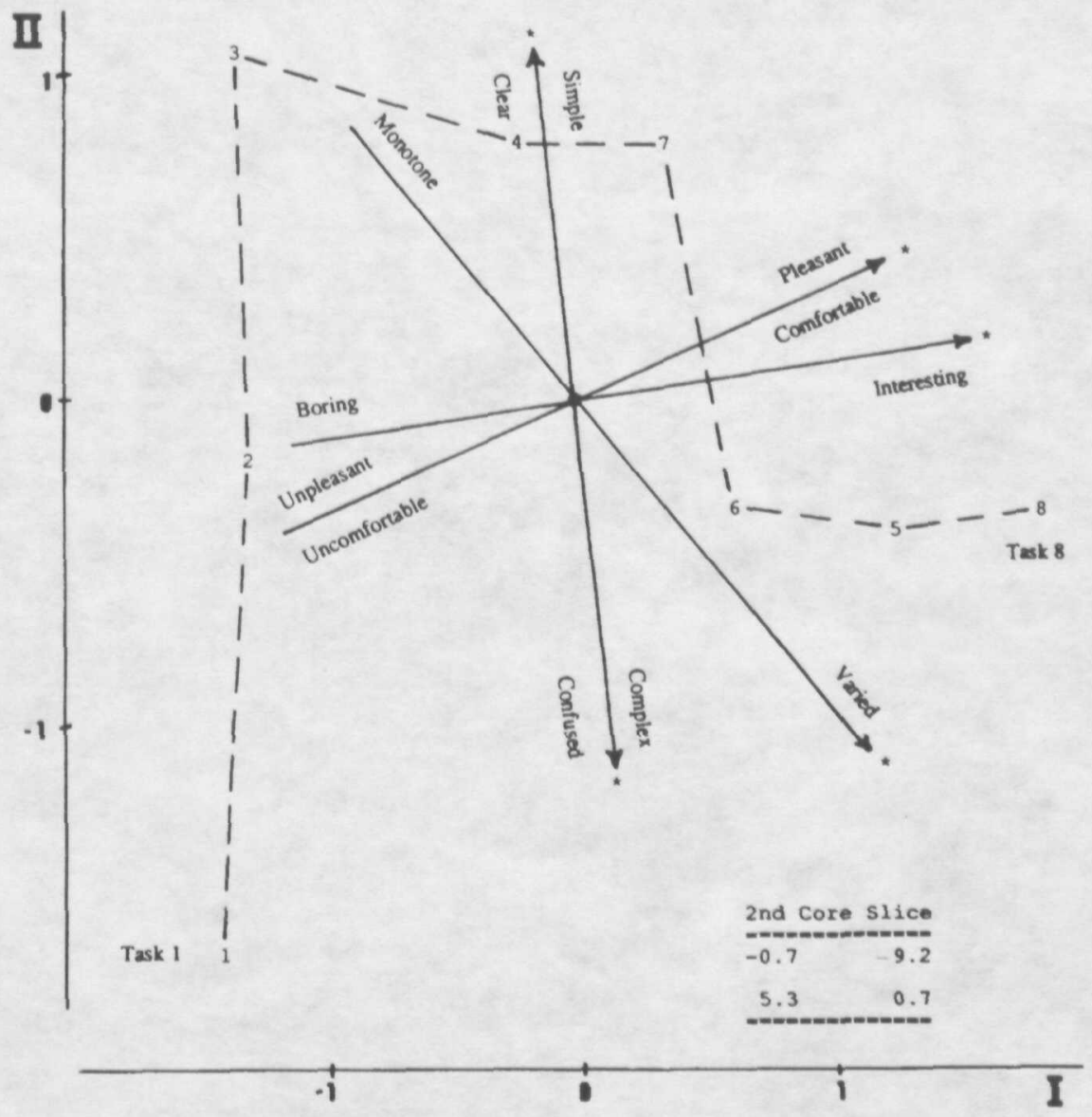

Figure 3.

Joint Plot of Scales and Tasks for Second Subject Type. 
So far we have dealt with the data on varying degrees of aggregation, and it became apparent that there were many differences between individuals. An attractive way of further investigating these differences is to compute, on the basis of the three-mode parameters, scores for each subject/task unit on the two scale dimensions, $\mathrm{S} 1$ and S2. By using the restricted number of parameters of the model these scores are more regular than those from a standard (two-mode) component analysis. Of course, for ill-fitting boys (see Table 5) the behavior can only be described approximately, especially if a separate analysis of their data would show a very different pattern. The fit for such a subject reflects only that part of his data that are in agreement with the common structure.

Low fit in the joint analysis does not necessarily mean that there is little structure in a boy's data. For instance, individual principal component solutions of boys 20 and 28 (boys with low fit [ $22 \%$ and $7 \%$, respectively] compared to the average of $44 \%$ ) show that $91 \%$ and $88 \%$ of their data can be fitted by two components, compared to a maximum of $78 \%$ for a random subject. Thus there is at least some system in the way they score the rating scales. This need not always be the case, as can be seen in a study on implicit theories of personality (Van der Kloot \& Kroonenberg, 1982). These authors showed that if a subject fitted badly in the overall solution, the fit also was low in the individual solution.

Table 6

\section{Order of Tasks on First Scale Component}

\begin{tabular}{clrr}
\hline \multicolumn{1}{c}{ Orders } & \multicolumn{1}{c}{ Subjects } & Frequency & \#Swaps \\
\hline $1,2,3,4,6,7,5,8$ & $1,2,8,9,11, \underline{13}, 16,17,19,25,29,32$ & 12 & 0 \\
$1,2,3,4,7,6,5,8$ & $4,21,26$ & 3 & 1 \\
$1,2,3,6,4,7,5,8$ & 3,7 & 2 & 1 \\
$1,2,3,6,4,5,7,8$ & $\mathbf{2 2}$ & 1 & 2 \\
$1,2,6,3,4,5,8,7$ & 5,18 & 2 & 2 \\
$1,2,6,8,5,4,3,7$ & $\mathbf{1 2 ,} \underline{24}, 31$ & 3 & 10 \\
& & & \\
$3,2,1,4,7,6,5,8$ & $10,23,27, \underline{30}$ & 4 & 4 \\
$3,2,4,7,1,6,5,8$ & $\mathbf{6}, \underline{4}$ & 2 & 5 \\
$3,2,4,7,5,6,8,1$ & $\mathbf{1 5}$ & 1 & 10 \\
$3,4,7,5,2,8,6,1$ & $\mathbf{2 8}$ & 1 & 14 \\
$1,6,8,5,2,4,7,3$ & $\mathbf{2 0}$ & 1 & 14 \\
\hline
\end{tabular}

Note. Bold subjects have a fit within the range of the random subjects. Underlined subjects have a fit in the range $.23<$ fit $<.30$. 
The individual characteristics of boys with a fit above .30 (i.e. well above the best random fit) are presented in two figures. The Complexity curves (Figure 4) show generally well-behaved subjects with mostly the empirical order for the tasks. Tasks 1,2, and 3 do not differ much in Complexity, and 6 precedes 7 for about half of the boys; Task 5 is (generally) somewhat more difficult than 6 and 7 , and somewhat less so than Task 8 (for details on all different orders, see Table 6). The extreme curves have been connected to show the different patterns. Some boys (e.g., 3, 5, I, V) find earlier tasks increasingly complex until Task 4, while the later tasks do not differ very much. At the other end, some boys (e.g., 4, A, N, Q, R, and T) find the first three tasks easy (in fact, A, N, R, and T paradoxically see the first three tasks slightly decreasing in difficulty), and from Task 4 on, the difficulty increases rapidly, and virtually monotonically. The other boys take up intermediary positions between the extremes.

With respect to Figure 5, the Interesting/Pleasantness aspects of the tasks are judged in many different ways. One subset of boys (e.g., 3, H, L, T, and W) has clear single-peaked functions with respect to this Scale component, while a second subset (e.g., 5 and $\mathrm{V}$ ) has virtually monotonically decreasing curves. A third subset (e.g., O, B, J, and R) has largely increasing curves.

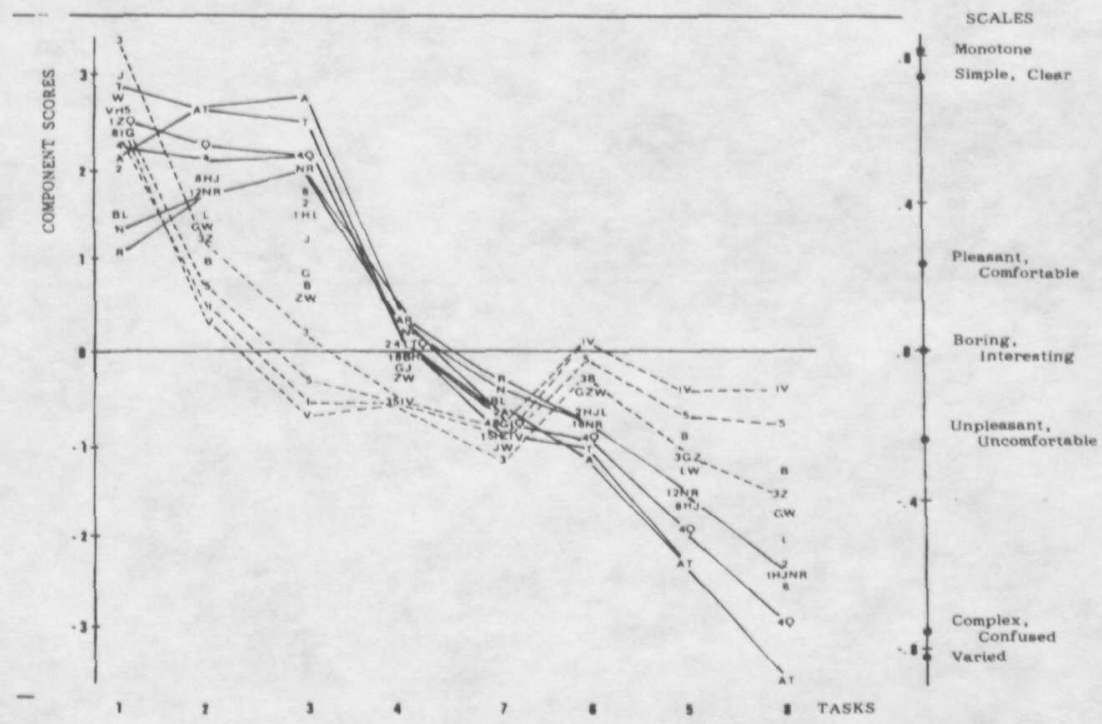

\section{Figure 4.}

Scores of Task/Subject Combinations on First Scale Component versus Empirical Task Order. Note: Similar profiles are connected by similar lines. Labels in the plot refer to the subjects of Table 5. Scale loadings are taken from Table 4. 


\section{P. Kroonenberg and C. Snyder}

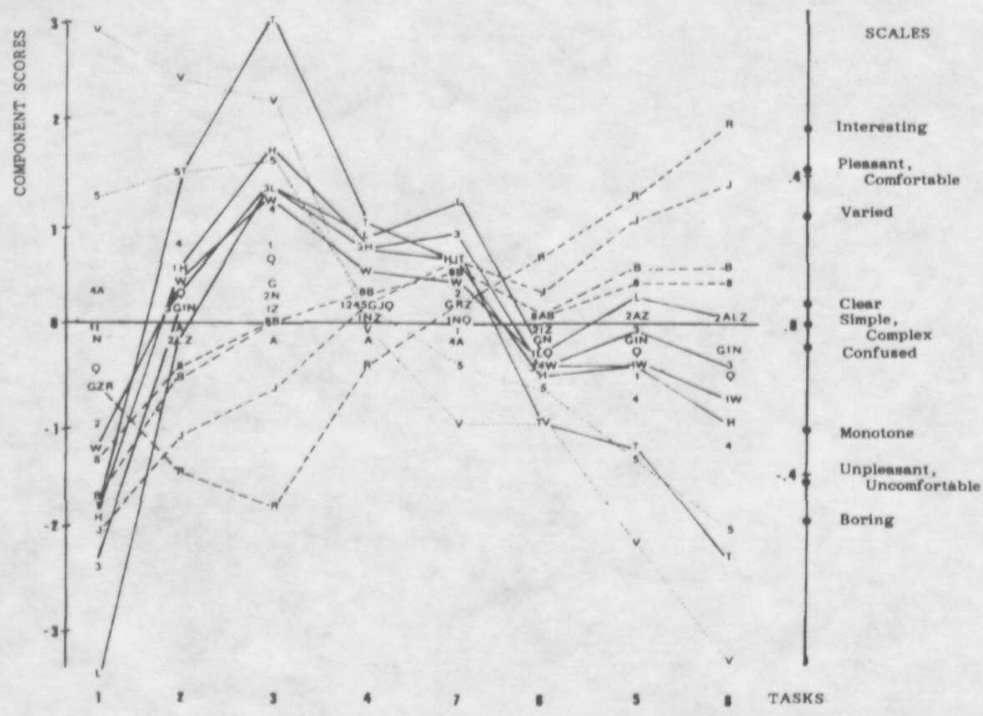

Figure 5.

Scores of Task/Subject Combinations on Second Scale Component versus Empirical Task Order. Note: Similar profiles are connected by similar lines. Labels in the plot refer to the subjects of Table 5. Scale loadings are taken from Table 4.

\section{Conclusions and Implications}

At the highest level of aggregation, given the principal component analyses of the one-mode correlation matrices for this study and Eckblad's 1981(a) study, Eckblad's hypotheses seem tenable. The summary evidence from these studies supports the contention that the relational patterns of structural and affective judgments conform to an ordered configuration of the variable vectors and that this pattern results from the distribution of perceived information complexity in the problem solving tasks from which these judgments were evoked. Furthermore, when comparing the Eckblad results for the two age groups used in her studies, with corroborating evidence from the work of Bragg and Crozier (1974; see also Eckblad, 1980,pp. 11-12), which also employed different age groups, the angles between Pleasant, Interesting, and Complex appear meaningfully linked to developmental levels of information processing capacity.

There are two results in the correlative vector configuration for the boys sample which require further discussion. First, the pattern of vectors in this study (Figure 1) is much more vulnerable to a clustered dimensional interpretation than the 1981 configuration (see Eckblad, 1981a, her Figure 3, p. 22). Second, the position of Task 5 is not as predicted. Both of these results can be rationalized within Eckblad's theoretical framework, even though precise explanatory statements are well beyond this empirical investigation. 
As argued by Eckblad (1980), on evidence from Hutt, Forrest, and Newton (1976), younger subjects may be less able to differentiate between the terms Interesting and Pleasant. Thus, in this study of young adolescents, these semantically ambiguous variables may show up as minimally differentiated vectors against their covariation background. Figure 1 displays two clusters of vectors: Interesting-Boring, Comfortable-Uncomfortable, and Pleasant-Unpleasant as one (Affect), and Varied-Monotone, Complex-Simple, and Confused-Clear as the other (Structure of Task). The vectors group in this fashion primarily because the boys fail to differentiate the Interesting scale from the Pleasant scale. Those variables that relate curvilinearly to Complexity (i.e., the structural scales) group into the other; and within a cluster, the variables begin to fan out only slightly.

Although interpretively uncomfortable, the minimal differentiation of affect labels in this sample is consistent with Eckblad's developmental views on the affect spectrum. Affect and cognition in Eckblad's theory are two aspects of the same process, in this case, problem solving. Piaget's influence is apparent:

Affective life and cognitive life, then, are inseparable although distinct. They are inseparable because all interaction with the environment involves both a structuring and a valuation, but they are nonetheless distinct, since these two aspects of behaviour cannot be reduced to one another. Thus we could not reason, even in pure mathematics, without experiencing certain feelings, and conversely no affect can exist without a minimum of understanding or discrimination. (Piaget, 1950, pp. 5-6)

At the age of thirteen, the boys are only beginning to apply formal operations, enabling them to reflect on what and how they think (Piaget, 1981). Furthermore, since affective words emerge from a public language, tied as they are to cultural and social meanings rather than a private language which would be inextricably tied to personal meanings (cf. Wittgenstein, 1968), the labels for affective experiences are imprecise. If youths have limited information processing capacity to achieve valid symbolic representation and the symbols are hazy (public) reflections, then the affect structure can be expected to be less well defined in the younger sample.

The three-mode analysis probes deeper into the empirical foundations of Eckblad's theory, examining the nomothetic validity of the theoretical framework (see Snyder, Law, \& Hattie, 1984) in terms of each and every data point. The fit under this scrutiny loosens to expose the modeling discrepancies. Most studies stop at the higher aggregate levels and understandably so. The probe finds those framework gaps ordinarily covered over by the group and relational summaries of correlative analyses. Gaps exist in all theoretical frameworks: 
1. mivuriermery ariu $\cup$. misuer

I do know one fact about this system and about all the other systems of 'emotion' they are all, to some extent, wrong. The joy is to try to be a little less wrong next time around. (Mandler, 1975, p. 66, refering to his cognitive theory of emotion, which was developed independently of Eckblad's, but resembles it in many ways, particularly in his 1982 paper.)

Eckblad's theorizing lends itself to three-mode analysis. Her affect framework entails three-mode hypotheses, in that she has predictions about the behavior of variables within each of the classification sets and the relationships between these modes. She posits that scales, tasks, and individuals are all points in a common space defined by the joint attributes of these modes, which in this case represent the notion of assimilation resistance. AR is an interactive construct. An individual carries a personal assimilatory history and a set of common, but subjectively constructed judgment scales, into any encounter. Each event or object encountered presents a certain information load which must be assimilated by the individual in order for that individual to operate meaningfully within the context. Naturally any such encounter will be colored by the social and cultural influences that are part of an individual's self construct and the context itself, but the most immediate effects are some level of assimilation and subjective judgments (beliefs) about the experience. Eckblad formulates an unfolding model (see Coombs, 1964) to describe the joint effects. According to this model, the scales and individuals share the same metric space distributing themselves as ideal points across the AR range.

In this study the information load of the tasks is manipulated so that the order of tasks approximately maps a sufficient range of loads to provide different levels of resistance. Portrayed in the principal component analogue of unfolding, the joint plots (Figures 2 and 3 ) nicely display the intended results for two individual types (reflecting two possible sets of linear combinations). The Task $J$ scale and the Judgment I scale conform to the expected behavior of unfolded preference-type data, but the stability of the structure is hampered by the fact that Tasks 4,6 , and 7 contribute only about $3 \%$ combined to the $37 \%$ fit in the task mode, and Pleasant, Interesting, and Comfortable contribute only about $9 \%$ combined to the equivalent fit in the scales mode. A strict unfolding model would collapse under these stresses. Although the less constrained component analysis algorithm accommodates these problems, the overall fit suffers accordingly.

One problem may be the nature of the tasks and the procedural context. Either because it is an experiment in problem solving or because it is perceived as another school-like activity, the affect reporting may be constrained. Certainly very little of the overall variance is attributable to the affect terms; most arises from the structural side. This can be explained partly in terms of expectancies. Problem solving tasks are expected to range in complexity, but not necessarily pleasantness. It has been demonstrated that subjects' expectations about the 
experimental experience can influence their use of affect judgment scales (cf. Anderson \& Pennebaker, 1980), so the limited differentiable affect may reflect a self-fulfilling prophecy for young students. Even if present, however, this effect would merely dull the relationships.

A more important consideration is the extent to which the tasks can overcome prior expectancies and engage the subjects in the activity of solving the problems. This quality of the tasks relates to the differences in the terms Pleasant and Interesting. Pleasant refers most directly to internal sensations and is probably processed at a very low level. Interesting seems to correspond to higher levels of activity, that is, more information is assimilated from the environment (Pennebaker, 1980). It designates the "moving edge of assimilation" (Dember \& Earl, 1957), where tasks tax a subject's capacity but offer a chance of successful assimilation (Eckblad, 1981b, p. 94). If the difficulty of these tasks exceeds the assimilation capacity of the youth so that affect ratings reflect the development of schemes rather than the deeper extension and consolidation of thought, then the quality of Interestingness will not appear in the process and it will be indistinguishable from Pleasantness (Eckblad, 1981b). Methodologically, since the heterogeneity of adjective ideal points is essential in order to adequately define the task space in accordance with the model, the absence of interesting tasks will define a space that will reflect only those consistent dimensional preferences of individuals (see Coombs, 1964, p. 94) rather than the hypothesized spectrum. To the extent that this has occurred, the effect would account for the differences in fit between the two samples (Eckblad, 1981a, this study), but would not account totally for the imprecision of the model fit in both samples.

Overall, the most important problem, in our judgment, is the operationalization of AR. Because AR is a within-subject construct, dependent on the assimilation capacity of the individual and the information load of the event, information complexity is only one part of the notion and does not necessarily correspond to AR (Eckblad, 1981b, p. 82). Information complexity is a between-subjects construct (and this was the criterion used in the selection of the tasks; Eckblad, $1980 ; 1981 \mathrm{~b})$. The manipulation of information complexity is only an approximate translation of the AR construct. Comparing the results from Eckblad's 1981(a) and this study, the between-subjects ratings are not the same. Task 5 (or Tasks $4,6,7$ ) is (are) seen differently by this younger sample. In fact, even if we allow up to 2 swaps from the empirical order as indicative of structural homogeneity, only 17 of the 32 boys share a common view of the tasks. The scaling of the data was intended to neutralize assimilatory background differences, so these different views are different in terms of structural variables as well as affect. An improvement in the procedural interpretation of AR is necessary to sort out these concerns. 
r. Kroonenberg and C. Snyder

Even within the information processing literature, attempts to assess information complexity and processing capacity have not yielded objective, unambiguous results (Halford, 1986).

Therefore in the present state of knowledge it seems that any attempt to assess the information processing demand of a task must depend on [the experimenter's] judgment and on assumptions too numerous to be stated. (Halford, 1986, p. 7)

In response to these problems, Halford $(1982,1984,1986$, Halford \& Wilson, 1980 ) has proposed a category theory approach to cognitive processing and information utilization which assesses the minimum information required for a decision under the constraints of the conditions of performance. This directly and objectively addresses the interaction in a way that is quite compatible with the AR notion of Eckblad (perhaps because the two theories share their lineage with Piaget's contributions). Assimilation is analogous, in Halford's theory, to the assignment of an environment system (the tasks) to a symbol system (the representational function in cognitive processes). Symbolic processes comprise systems that must be consistent and structurally isomorphic with the environment system they represent, that is, all mappings of symbol elements into environmental elements must be invariant throughout the system (Halford \& Wilson, 1980). Different forms of mappings define different levels of system complexity (assimilation capacity). When the symbolic system is not consistent with an environment system, then the symbolic system must accommodate (in Piaget's terms) and the (possibly temporary) disequilibrium (AR in Eckblad's terms) will mediate the concomitant affect. The advantage of Halford's theory lies in his mathematically rigorous criterion for the determination of the amount of information needed to construct a problem solution. This can be applied to the assessment or development of graded tasks such that AR levels are defined in terms of the logically minimum information processing demands presented by a task rather than empirical difficulty, which is subject to sample fluctuations. Without this more precise procedural translation of the AR concept, incorrect hypotheses associated with Eckblad's theory cannot be discerned from procedural invalidities and idiosyncratic sample variations. However, given the general validity of the theory's propositions, the theory cannot be ignored.

\section{References}

Anderson, D. B., \& Pennebaker, J. W. (1980). Pain and pleasure: Alternative interpretations of identical stimulation. European Journal of Social Psychology, 10, 207-212.

Berlyne, D. E. (1971). Aesthetics and psychobiology. New York: Appleton-Century-Crofts. Berlyne, D. E. (Ed.). (1974). Studies in the new experimental aesthetics: Steps toward an objective psychology of aesthetic appreciation. New York: Wiley. 
Bragg, D. W. E., \& Crozier, J. B. (1974). The development with age of verbal and exploratory responses to sound sequences varying in uncertainty level. In D. E. Berlyne (Ed.), Siudies in the new experimental aesthetics: Steps toward an objective psychology of aesthetic appreciation. New York: Wiley.

Coombs, C. H. (1964). A theory of data. New York: Wiley. (Reprinted, Ann Arbor, MI: Mathesis Press, 1976.)

Coombs, C. H., \& Kao, R. C. (1960). On a connection between component analysis and multidimensional unfolding. Psychometrika, 25, 219-231.

Dember, W. N., \& Earl, R. W. (1957). Analysis of exploratory, manipulatory, and curiosity behaviors. Psychological Review, 64, 91-96.

Dixon, W. J. (Ed.). (1981). BMDP statistical software 1981. Berkeley, CA: University of California Press.

Eckblad, G. (1978). Stimulus seeking and curiosity: A review and re-interpretation of studies in the Berlyne tradition. (Unpublished Report No. 1), Bergen, Norway: Institute of Psychology, University of Bergen.

Eckblad, G. (1980). The curvex: Simple order structure revealed in ratings of complexity, interestingness, and pleasantness. Scandinavian Journal of Psychology, 21, 1-16.

Eckblad, G. (1981a). Assimilation resistance and affective response in problem solving. Scandinavian Journal of Psychology, 22, 1-16.

Eckblad, G. (198Ib). Scheme theory: A conceptual framework for cognitive-motivational processes. London: Academic Press.

Efron, B. (1982). The jackknife, the bootstrap, and other resampling plans. SIAM Monograph, 38. (CMBS-NSF)

Halford, G. S. (1982). The development of thought. Hillsdale, NJ: Lawrence Erlbaum Associates.

Halford, G. S. (1984). Can young children integrate premises in transitivity and serial order tasks? Cognitive Psychology, 16, 65-93.

Halford, G. S. (1986). Children's utilization of information: A hasic factor in cognitive development. Unpublished manuscript, Department of Psychology, University of Queensland, St. Lucia, Australia.

Halford, G. S., \& Wilson, W. H. (1980). A category theory approach to cognitive development. Cognitive Psychology, 12, 356-411.

Harshman, R. A., \& Lundy, M. E. (1984a). The PARAFAC model for three-way factor analysis and multidimensional scaling. In H. G. Law, C. W. Snyder, Jr., J. A. Hattie, \& R. P. McDonald (Eds.), Research methods for multimode data analysis (pp. 122-215). New York: Praeger.

Harshman, R. A., \& Lundy, M. E. (1984b). Data preprocessing and the extended PARAFAC model. In H. G. Law, C. W. Snyder, Jr., J. A. Hattie, \& R. P. McDonald (Eds.), Research methods for multimode data analysis (pp. 216-284). New York: Praeger.

Humphreys, L. G., \& Montanelli, R. G., Jr. (1975). An investigation of the parallel analysis criterion for determining the number of common factors. Multivariate Behavioral Research, 10, 193-205.

Hutt, C., Forrest, B., \& Newton, J. (1976). The visual preferences of children. Journal of Child Psychology and Psychiatry, 17, 63-68.

Kroonenberg, P. M. (1983a). Three-mode principal component analysis: Theory and applications. Leiden, The Netherlands: DSWO Press.

Kroonenberg, P. M. (1983b). Annotated bibliography of three-mode factor analysis. British Journal of Mathematical and Statistical Psychology, 36, 81-113. 
r. nroonenoerg ana ᄂ. snyaer

Kroonenberg, P. M. (1984). Three-mode principal component analysis: Illustrated with an example from attachment theory. In H. G. Law, C. W. Snyder, Jr., J. A. Hattie, \& R. P. McDonald (Eds.), Research methods for multimode data analysis (pp. 64-103). New York: Praeger.

Kroonenberg, P. M. ,\& Brouwer, P. (1985a). User's guide to TUCKALS2. (WEP Reeks, WR 85-

09-RP). Leiden, The Netherlands: Department of Education, Leiden University.

Kroonenberg, P. M. ,\& Brouwer, P. (1985b). User's guide to TUCKALS3 (WEP Reeks, WR 85-

12-RP). Leiden, The Netherlands: Department of Education, Leiden University.

Kroonenberg, P. M., \& de Leeuw, J. (1980). Principal component analysis of three-mode data

by means of alternating least squates algorithms. Psychometrika, 45, 69-97.

Kroonenberg, P. M., Snyder, C. W., Jr., \& Law, H. G. (1984). Bibliography. In H. G. Law,

C. W. Snyder, Jr., J. A. Hattie, \& R. P. McDonald (Eds.), Research methods for multimode data analysis (pp. 643-662). New York: Praeger.

Levin, J. (1965). Three-mode factor analysis. Psychological Bulletin, 64, 442-452.

Lundy, M. E., Harshman, R. A., \& Kruskal, J. B. (1985, June). A two-stage procedure incorporating good features of both trilinear and quadrilinear methods. Paper presented at the 1985 meeting of the Classification Society (North American Branch). St. John's, Newfoundland.

Mandler, G. (1975). Mind and emotion. New York: Wiley.

Mandler, G. (1982). The structure of value: Accounting for taste. In M. S. Clark, \& S. T. Fiske (Eds.), Affect and cognition: The seventeenth annual Carnegie symposium on cognition (pp. 3-36). Hillsdale, NJ.: Erlbaum.

McDonald, R. P. (1984). The invariant factors model for multimode data. In H. G. Law, C. W. Snyder, Jr., J. A. Hattie, \& R. P. McDonald, Research methods for multimode data analysis (pp. 285-307). New York: Praeger.

Pennebaker, J. W. (1980). Perceptual and environmental determinants of coughing. Basic and Applied Social Psychology, I, 83-91.

Piaget, J. (1950). The psychology of intelligence. London: Routledge \& Kegan Paul.

Piaget, J. (1981). Intelligence and affectivity: Their relationship during child development (T.

A. Brown, \& C. E. Kaegi, Trans. and Eds.). Palo Alto, CA: Annual Reviews.

Snyder, C. W., Jr. (1988). Multimode factor analysis. In J. R. Nesselroade, \& R. B. Cattell (Eds.), Handbook of multivariate experimental psychology (2nd ed.). New York: Plenum.

Snyder, C. W., Jr., Law, H. G., \& Hattie, J. A. (1984). Overview of multimode analytic methods. In H. G. Law, C. W. Snyder, Jr., J. A. Hattie, \& R. P. McDonald (Eds.), Research methods for multimode data analysis (pp. 2-35). New York: Praeger.

Tucker, L. R. (1966). Some mathematical notes on three-mode factor analysis. Psychometrika, $31,279-311$.

Tucker, L. R. (1972). Relations between multidimensional scaling and three-mode factor analysis. Psychometrika, 37, 3-27.

Van der Kloot, W. A., \& Kroonenberg, P. M. (1982). Group and individual implicit theories of personality: An application of three-mode principal component analysis. Multivariate Behavioral Research, 17, 471-492.

Van der Kloot, W. A., Kroonenberg, P. M., \& Bakker, D. (1985). Implicit theories of personality: further evidence of extreme response style. Multivariate Behavioral Research, 20, 369-387.

Visser, R. A. (1985). Analysis of longitudinal data in behavioural and social research. Leiden, The Netherlands: DSWO Press.

Wittengenstein, L. (1968). Philosophical investigations. New York: Macmillan. 


\title{
Model Modification in Covariance Structure Analysis: Application of the Expected Parameter Change Statistic
}

\author{
David Kaplan \\ Department of Educational Studies \\ University of Delaware
}

This paper examines the problem of model modification in covariance structure analysis. Two methods of model modification are studied: the Modification Index (MI) which suggests modifications based on the largest drop in the overall value of the test statistic, and the Expected Parameter Change Statistic (EPC) which suggests modifications based on the removal of large and interesting specifications errors. Following a detailed discussion of the theory behind the MI and EPC, these methods are studied and applied to two specifications of the Wisconsin status attainment model. Additionally, a standardized version of the EPC statistic (SEPC) is proposed and applied to one of these models. Results indicate that the MI tends to suggest freeing substantively implausible parameters. The EPC and SEPC, by contrast, suggest freeing substantively interesting parameters. Results are discussed in terms of the practice of covariance structure modeling.

In the routine practice of structural equation modeling, a researcher may find that his/her model is not in agreement with the data as evidenced by standard statistical tests. Lack of agreement may be due to the fact that distributional assumptions have been violated (Boomsma, 1983; Muthen \& Kaplan, 1985; in press), problems of missing data (Muthen, Kaplan, \& Hollis, 1987), and/or model misspecification. Also, it may be the case that the test statistic is overly sensitive to the size of the sample, such that trivial misspecifications are being detected. The problem of strong sensitivity to sample size is particularly relevant for models which are estimated on large samples.

The relationship between sample size and size of misspecification implies that the power of the test statistic needs to be considered. Power refers to the probability of rejection of the null hypothesis implied by the model when the null hypothesis is false. Assessing power allows us to determine the extent to which the test statistic is strongly sensitive to sample size. Recent theoretical developments by Satorra and Saris (1985) make it possible to calculate power

The author would like to thank Bengt Muthen, Albert Satorra, Peter Bentler, and James Crouse for their valuable comments on an earlier version of this paper. Requests for reprints should be sent to the author, Department of Educational Studies, University of Delaware, Newark, DE 19716. 